\title{
Formation of lower-hybrid solitary structures by modulational interaction between lower-hybrid and dispersive Alfvén waves
}

\author{
J. O. Hall ${ }^{1,{ }^{*}}$, G. Stenberg ${ }^{1}$, A. I. Eriksson ${ }^{1}$, and M. André ${ }^{1}$ \\ ${ }^{1}$ Swedish Institute of Space Physics, Uppsala, Sweden \\ * present address: Swedish Defence Research Agency, Stockholm, Sweden
}

Received: 4 June 2008 - Revised: 2 February 2009 - Accepted: 4 February 2009 - Published: 2 March 2009

\begin{abstract}
We investigate the possibility that lower-hybrid solitary structures (LHSS), which are frequently observed in the Earth's ionosphere and magnetosphere, are formed as a result of a modulational interaction between lower-hybrid and dispersive Alfvén waves of initially small amplitude. A large amplitude lower-hybrid pump wave can excite density structures with length scales transverse to the geomagnetic field of the order of the ion gyroradius via a modulational instability. The structure formation in the nonlinear stage of the instability is investigated by numerical solutions of the governing equations, using plasma parameters relevant for LHSS observations in the upper ionosphere and in the magnetosphere. The numerical solutions reveal that the lower-hybrid waves become self-localized inside cylindrically symmetric (with respect to the ambient magnetic field) density cavities, in qualitative agreement with observations. Our model includes thermal electron effects but shows no stabilization at the ion sound gyroradius, suggesting that any preference of observed LHSS for that perpendicular scale likely is due to processes arresting the cavity collapse.
\end{abstract}

Keywords. Space plasma physics (Nonlinear phenomena; Numerical simulation studies; Wave-wave interactions)

\section{Introduction}

Spacecraft measurements have shown that localized bursts of lower hybrid (LH) waves are common events in the ionosphere (LaBelle et al., 1986; Kintner et al., 1992; Eriksson et al., 1994; Pécseli et al., 1996; Bonnell et al., 1998) and in the magnetosphere (Tjulin et al., 2003; Tjulin et al., 2004a). It has been confirmed that the wave activity coincides with density depletions that are elongated along the geomagnetic

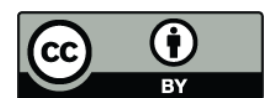

Correspondence to: J. O. Hall (jan-ove.hall@foi.se) field lines. These elongated density structures with localized waves in the LH frequency range are termed LH cavities (LHC) or LH solitary structures (LHSS). The relative decrease in the plasma number density inside the cavities is typically a few percent at altitudes of 1500-13000 km (Dovner et al., 1997; Tjulin et al., 2004a) to a few tens of percent below 1500 km (Kintner et al., 1992; Knudsen et al., 2004) and above $20000 \mathrm{~km}$ (Tjulin et al., 2004a). The perpendicular width is observed to be of the order of the ion gyroradius and to scale with this parameter better than with the electron inertial length (Knudsen et al., 2004). The parallel dimension of the density cavity is estimated to be several order of magnitudes larger (Kintner et al., 1992; Pécseli et al., 1996; Tjulin et al., 2004b), and such elongated LHSS have indeed been directly observed by a two-payload sounding rocket experiment (Knudsen et al., 1999).

The wave front of the localized waves is observed to rotate around the ambient magnetic field (Pinçon et al., 1997; Bonnell et al., 1998; Tjulin et al., 2003) in a left-handed (right-handed) sense for wave frequencies below (above) the LH frequency $\omega_{L H}=\omega_{p i} /\left(1+\omega_{p e}^{2} / \omega_{c e}^{2}\right)^{1 / 2}$, where $\omega_{p e}=\left(4 \pi n_{0} e^{2} / m_{e}\right)^{1 / 2}$ and $\omega_{p i}=\left(4 \pi n_{0} e^{2} / m_{i}\right)^{1 / 2}$ are the electron and ion plasma frequencies, respectively, $\omega_{c e}=e B_{0} /\left(m_{e} c\right)$ is the electron gyrofrequency, $n_{0}$ is the background plasma number density, $e$ is the magnitude of the electron charge, $B_{0}$ is the strength of the external magnetic field, $c$ is the speed of light in vacuum, and $m_{e}\left(m_{i}\right)$ is the electron (ion) mass. Further, the LHSS are found to be a source of transverse ion acceleration (Lynch et al., 1999; Knudsen et al., 2004). The literature on LHSS was reviewed by Schuck et al. (2003).

The LHSS are always observed to be immersed in nonlocalized wave activity with wave frequencies $\sim \omega_{L H}$. The non-localized waves are collectively referred to as "hiss" (Sazhin et al., 1993). The electric field associated with hiss emissions is strongest for wave frequencies above $\omega_{L H}$, but electromagnetic wave activity below $\omega_{L H}$ is also observed.

Published by Copernicus Publications on behalf of the European Geosciences Union. 
Table 1. Plasma parameters and derived dimensionless quantities relevant for LHSS observations in the topside ionosphere (Eriksson et al., 1994, Freja) and magnetosphere (Tjulin et al., 2004a, Cluster). Magnetosphere temperatures are assumed values. The values for Freja are used in the numerical study (Sect. 3).

\begin{tabular}{lrrl}
\hline Parameter & Freja & Cluster & Unit \\
\hline Plasma density $n_{0}$ & 900 & 10 & $\mathrm{~cm}^{-3}$ \\
Magnetic field $B_{0}$ & 250 & 3 & $\mathrm{mG}$ \\
Ion temperature $T_{i}$ & 2400 & 10000 & $\mathrm{~K}$ \\
Electron temperature $T_{e}$ & 3000 & 10000 & $\mathrm{~K}$ \\
Ion mass $m_{i}$ & $16 \times 1836$ & 1836 & $m_{e}$ \\
Plasma beta $\beta$ & $1.5 \times 10^{-7}$ & $4 \times 10^{-5}$ & - \\
Mass ratio $m_{e} / m_{i}$ & $3.4 \times 10^{-5}$ & $5.4 \times 10^{-4}$ & - \\
$\omega_{p e}^{2} /\left(\omega_{c e} \omega_{c i}\right)$ & $4 \times 10^{3}$ & $2 \times 10^{4}$ & - \\
\hline
\end{tabular}

The wave activity with wave frequencies above but close to $\omega_{L H}$ is mainly attributed to electrostatic $\mathrm{LH}$ waves. The structure of the electric field localized inside the cavities is well described by linear eigenmode analysis of electrostatic (Seyler, 1994; Schuck et al., 1998) or electromagnetic (Hall et al., 2004; Hall, 2004) waves in a density depletion. However, the low-frequency dynamics and the formation of LHSS are not yet well understood. In this study, we investigate a possible scenario for LHSS formation, using a fluid description of interaction between lower-hybrid (LH) waves in the non-localized hiss activity and dispersive Alfvén (DA) waves. Due to a parametric instability, the initially small amplitude DA waves can grow and eventually form nonlinear structures. We wish to point out that this model concerns the initial formation of the LHSS, and that other mechanisms may be important for their later maintenance and decay (Robinson, 1991). In particular, ion kinetics leading to ion heating and expulsion may be important at later stages, as described by Knudsen et al. (2004).

There has been a great deal of interest in understanding the nonlinear interaction between the LH and DA waves, and efforts to describe the modulational interaction between the two modes have been made (Shapiro, 1998; Shukla and Stenflo, 1999; Shapiro et al., 2003; Ücer and Shapiro, 2004; Hall et al., 2005; Ücer and Shapiro, 2005). In the treatments by Shapiro (1998), Shapiro et al. (2003), Ücer and Shapiro (2004), and Ücer and Shapiro (2005), the dispersion of the Alfvén wave was considered to be exclusively due to finite electron inertia. The perpendicular dispersion length is consequently equal to the collisionless electron skin depth $\lambda_{e}=c / \omega_{p e}$. In an extremely low- $\beta$ plasma, the effect of parallel electron kinetics give rise to additional perpendicular dispersion at perpendicular length scales much smaller than $\lambda_{e}$. The relevant dispersion length in this short wavelength regime is the ion gyroradius at the electron temperature $\rho_{s}=\left(T_{e} / m_{i}\right)^{1 / 2} \ll \lambda_{e}$, where $T_{e}$ is the electron temperature. The treatments by Shukla and Stenflo (1999) and Hall et al. (2005) include effects due to finite electron inertia as well as effects due to parallel electron kinetics.

The plasma $\beta$ is extremely low in all plasmas where LHSS have been observed, in the ionosphere (Schuck et al., 2003) and (with less margin) in the magnetosphere (Tjulin et al., 2004a). This means that parallel electron kinetics effects, which give rise to dispersion on the $\rho_{s}$ scale, are potentially more important than effects of electron inertia, depending on which length scales are excited. That parallel electron kinetics effects indeed are important for LHSS formation is also indicated by the observation of Knudsen et al. (2004) that the LHSS transverse size appears to scale better with $\rho_{s}$ than with $\lambda_{e}$. The purpose of the present paper is to include such effects in realistic simulations, as this has not been done in previous papers on LHSS formation via DA/LH wave interactions (Shapiro, 1998; Shapiro et al., 2003; Ücer and Shapiro, 2004, 2005) or LH wave interactions with other wave modes (Seyler, 1994).

The DA waves are low-frequency waves in comparison to the ion gyrofrequency $\omega_{c i}=e B_{0} /\left(m_{i} c\right)$ and accompany a quasineutral density perturbation and a sheared magnetic field. The LH waves are sensitive to gradients in the plasma density and the low-frequency DA perturbations can therefore modulate the LH waves. On the other hand, the Reynolds stress of the LH waves couples back to the lowfrequency perturbations and modifies the DA waves. As a result of this interaction, a finite amplitude LH wave can be modulational unstable with respect to low-frequency DA perturbations. Hall et al. (2005) could show that this model predicts excitation of filamentary density structures with perpendicular width of the order of the ion gyroradius via a modified decay instability. In order to investigate the structure formation in the nonlinear stage of the instability, we present numerical solutions of the fully nonlinear three-dimensional equations of Hall et al. (2005). Starting with a large amplitude plane LH wave, the numerical solutions show that cylindrical density cavities are formed. The LH wave field becomes self-localized inside the cavities during the formation process. Further, the wave front of the localized wave rotates in a left-handed sense. These properties are in qualitative agreement with observations of LHSS and our investigation shows that the considered wave interaction can be important for the formation of LHSS.

\section{Basic equations}

In this study, we will consider plasma parameters typical of the conditions in the topside ionosphere seen at LHSS locations by the Freja satellite (Eriksson et al., 1994), which has contributed the bulk of all LHSS observations (Schuck et al., 2003). We list these parameters in Table 1, together with similar parameters for the LHSS observations in the magnetosphere (Tjulin et al., 2003). For the ionospheric observations, we assume an oxygen plasma, while hydrogen is 
assumed for the magnetospheric case. As can be seen from the table, we in both cases have that the ratio of kinetic to magnetic pressure, i.e. the plasma $\beta \equiv 8 \pi n_{0} T_{e} / B_{0}^{2}$ is well below the mass ratio $m_{e} / m_{i}$, and that $\omega_{p e}^{2} /\left(\omega_{c e} \omega_{c i}\right) \gg 1$. We will study LH waves with a wave number on the same order as the size of the density fluctuation, i.e. the perpendicular wavelength of the DA, so that $k_{L H} / k_{A} \ll \omega_{p e}^{2} /\left(\omega_{c e} \omega_{c i}\right)$. In these limits, the three-dimensional dynamics of nonlinearly coupled LH and DA waves propagating obliquely to an external magnetic field $\boldsymbol{B}_{0}=B_{0} \hat{z}$ described by (Hall et al., 2005) are described by the simplified equations

$$
\begin{aligned}
& \mathcal{L}_{L} \phi_{\mathrm{L}}=i \frac{\omega_{L H}}{\omega_{c i}}\left(\nabla_{\perp} \phi_{\mathrm{L}} \times \nabla_{\perp} \eta\right)_{z}, \\
& \begin{aligned}
d_{t}\left(1-\lambda_{e}^{2} \nabla_{\perp}^{2}\right) A_{z} & +c \partial_{z} \phi_{\mathrm{A}}-c \rho_{s}^{2} d_{z} \nabla_{\perp}^{2} \phi_{\mathrm{A}} \\
& =i \frac{c^{2}}{B_{0} \omega_{L H}} \frac{\partial}{\partial z}\left(\nabla_{\perp} \phi_{L}^{*} \times \nabla_{\perp} \phi_{L}\right)_{z},
\end{aligned}
\end{aligned}
$$

and

$d_{t} \nabla_{\perp}^{2} \phi_{\mathrm{A}}+\frac{v_{\mathrm{A}}^{2}}{c} d_{z} \nabla_{\perp}^{2} A_{z}=0$

where $v_{A}=B_{0} / \sqrt{4 \pi n_{0} m_{i}}$ is the Alfvén speed. Equation (1) describes the evolution of the LH wave, where $\phi_{L}(\boldsymbol{x}, t)$ is an envelope function with slow temporal evolution, viz., $\left|\partial_{t} \phi_{L}\right| \ll \omega_{L H}\left|\phi_{L}\right|$. The real valued LH wave potential is $\phi_{L H}=\phi_{L} \exp \left(-i \omega_{L H} t\right)+$ c.c., where c.c. symbolizes the complex conjugate. The linear dispersion of the LH waves is described by $\mathcal{L}_{L}=-\left(2 i / \omega_{L H}\right) \partial_{t} \nabla^{2}-\rho_{T}^{2} \nabla_{\perp}^{4}+\left(m_{i} / m_{e}\right) \partial_{z}^{2}, \quad$ where $\rho_{T}=\left\{3 T_{i} /\left(\omega_{L H}^{2} m_{i}\right)+2 T_{e} \omega_{p e}^{2} /\left[\left(\omega_{c e}^{2} m_{e}\right)\left(\omega_{p e}^{2}+\omega_{c e}^{2}\right)\right]\right\}^{1 / 2}$ is the thermal dispersion length and $T_{i}$ is the ion temperature. Equations (2) and (3) describe the DA wave, where $\phi_{A}$ and $A_{z}$ are the DA wave potential and the parallel (to $\boldsymbol{B}_{0}$ ) component of the vector potential, respectively. The electromagnetic fields are $\boldsymbol{B}_{\perp}=\nabla_{\perp} A_{z} \times \hat{z}$ and $\boldsymbol{E}_{A}=-\nabla \phi_{A}-\hat{z} c^{-1} \partial_{t} A_{z}$. The differential operators $d_{t}=\partial_{t}+\left(c / B_{0}\right) \hat{z} \times \nabla \phi_{A} \cdot \nabla$ and $d_{z}=\partial_{z}-\left(1 / B_{0}\right) \hat{z} \times \nabla A_{z} \cdot \nabla$ include self-interaction nonlinearities due to convection and field line bending, respectively. The DA wave accompanies a quasineutral density perturbation $n_{A} / n_{0} \equiv \eta=\left[c /\left(B_{0} \omega_{c i}\right)\right] \nabla_{\perp}^{2} \phi_{A}$, which arises from the ion polarization drift. The density perturbation modulate the LH waves, which is described by the nonlinear term in the right-hand side of Eq. (1). On the other hand, the Reynolds stress of the LH waves give rise to a ponderomotive force which drives the DA waves. The nonlinear driver of DA waves is accounted for by the nonlinear term in the right-hand side of Eq. (2).

In the linear approximation, Eq. (1) can be Fourier transformed to give the approximate $\mathrm{LH}$ dispersion relation

$$
\omega_{\boldsymbol{k}}=\omega_{L H}+\delta \omega_{\boldsymbol{k}} \equiv \omega_{L H}\left(1+\frac{1}{2} k_{\perp}^{2} \rho_{T}^{2}+\frac{1}{2} \frac{k_{z}^{2}}{k^{2}} \frac{m_{i}}{m_{e}}\right),
$$

where $\omega_{\boldsymbol{k}}$ is the LH wave frequency, $\delta \omega_{\boldsymbol{k}}$ is a linear frequency shift due to dispersion, and $k_{z}$ is the parallel component of the LH wavevector $\boldsymbol{k}=\boldsymbol{k}_{\perp}+k_{z} \hat{\boldsymbol{z}}$. Equation (4) describes accurately the dispersion of linear LH waves for $\rho_{i}<2 \pi / k_{\perp}<\lambda_{e}$, where $\rho_{i}=\left(T_{i} / m_{i}\right)^{1 / 2}$ is the ion gyroradius. Similarly, for small amplitudes, Eqs. (2) and (3) describe linear DA waves. We find the dispersion relation

$\Omega_{q}=\left[\frac{q_{z}^{2} v_{A}^{2}}{1+q_{\perp}^{2} \lambda_{e}^{2}}\left(1+q_{\perp}^{2} \rho_{S}^{2}\right)\right]^{1 / 2}$,

where $\Omega_{q}$ is the DA wave frequency and $q_{z}$ is the parallel component of the DA wavevector $\boldsymbol{q}=\boldsymbol{q}_{\perp}+q_{z} \hat{z}$. For $q_{\perp} \lambda_{e} \sim 1$, Eq. (5) describes dispersive inertial Alfvén waves for which $\Omega_{q} \approx q_{z} v_{A} /\left(1+q_{\perp}^{2} \lambda_{e}^{2}\right)^{1 / 2}$. On the other hand, for $q_{\perp} \lambda_{e} \gg 1$ and significant $q_{\perp} \rho_{S}$, Eq. (5) reduces to $\Omega_{q}=\left(q_{z} / q_{\perp}\right)\left(\omega_{c e} \omega_{c i}\right)^{1 / 2}\left(1+q_{\perp}^{2} \rho_{S}^{2}\right)^{1 / 2}$, which describes the electrostatic limit of the DA wave. This wave mode is known as the modified convective cell (Shukla et al., 1985).

\section{Evolution of the initial parametric instability}

In this section we discuss the stability of LH waves with respect to small amplitude DA wave perturbations. Here, we model the electrostatic hiss by a single finite amplitude monochromatic LH pump wave and effects due to the finite bandwidth of the ambient wave spectrum are neglected. For this purpose, we express the electrostatic potential of the excited DA wave as $\phi_{A}=a \exp [i(\boldsymbol{q} \cdot \boldsymbol{x}-\Omega t)]+$ c.c., where $\Omega$ and $\boldsymbol{q}$ is the frequency and wavevector of the DA wave, respectively. The LH wave potential is decomposed into the pump wave and two sidebands, i.e.

$$
\begin{aligned}
\phi_{L}= & \phi_{0} e^{i\left(\boldsymbol{k} \cdot \boldsymbol{x}-\delta \omega_{\boldsymbol{k}} t\right)}+\phi_{+} e^{i\left[(\boldsymbol{k}+\boldsymbol{q}) \cdot \boldsymbol{x}-\left(\delta \omega_{\boldsymbol{k}}+\Omega\right) t\right]} \\
& +\phi_{-} e^{i\left[(\boldsymbol{k}-\boldsymbol{q}) \cdot \boldsymbol{x}-\left(\delta \omega_{\boldsymbol{k}}-\Omega\right) t\right]},
\end{aligned}
$$

where $\boldsymbol{k}\left(\delta \omega_{\boldsymbol{k}}\right)$ is the wavevector (linear frequency shift) of the LH pump wave and $\phi_{0}\left(\phi_{ \pm}\right)$is the electrostatic potential of the LH wave pump (sidebands). Thus, Eqs. (1)-(3) can be Fourier transformed and combined to yield the nonlinear dispersion relation (Hall et al., 2005)

$$
\begin{aligned}
1 & +\frac{\omega_{L H}}{8} \frac{\Omega_{\boldsymbol{q}}^{2}}{\Omega^{2}-\Omega_{\boldsymbol{q}}^{2}} \frac{\lambda_{e}^{2} q_{\perp}^{2}}{1+\rho_{s}^{2} q_{\perp}^{2}} \frac{E_{0}^{2}}{\bar{E}^{2}} \frac{(\boldsymbol{k} \times \boldsymbol{q})_{z}^{2}}{k^{2} q^{2}} \\
& \times\left[\frac{\alpha_{+}}{-\Omega+\omega_{\boldsymbol{k}+\boldsymbol{q}}-\omega_{\boldsymbol{k}}}+\frac{\alpha_{-}}{\Omega+\omega_{\boldsymbol{k}-\boldsymbol{q}}-\omega_{\boldsymbol{k}}}\right]=0,
\end{aligned}
$$

where $\bar{E}=\omega_{c e} m_{e} B_{0} /\left(\omega_{p e} m_{i}\right)$ and $\alpha_{ \pm}=q^{2} /(\boldsymbol{k} \pm \boldsymbol{q})^{2}$. Equation (7) describes a three-wave decay with $\Omega \approx \Omega_{q}$ as well as a modified decay instability. The later occur for a large amplitude LH pump wave. The decay spectrum predicted by Eq. (7) is discussed in some detail in Hall et al. (2005). Equation (7) has been solved numerically for a LH pump wave with $\boldsymbol{k}=2 \pi /\left(1.7 \rho_{s}\right) \hat{\boldsymbol{x}} \mathrm{m}^{-1}$ and $E_{0}=6 \mathrm{mV} / \mathrm{m}$, using the plasma parameters cited in Table 1 for Freja observations of 


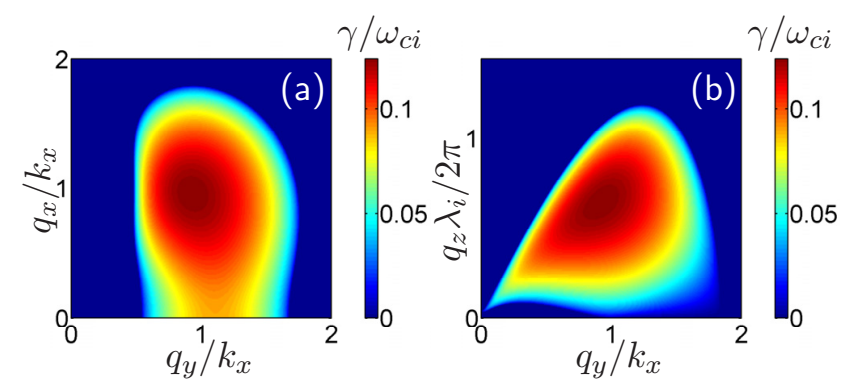

Fig. 1. The growth rate of the modified decay instability as a function of $\boldsymbol{q}$. Panel (a) shows $\gamma$ as a function of $\boldsymbol{q}_{\perp}$ for $q_{z}=\left(2 \pi / \lambda_{i}\right) \times 0.68$. Panel (b) shows $\gamma$ as a function of $q_{y}$ and $q_{z}$ for $q_{x}=k_{x}$.

LHSS in the Earth's upper ionosphere (Eriksson et al., 1994). As can be seen from the dimensionless numbers in the table, this regime applies also to magnetospheric LHSS observations.

Figure 1 shows $\gamma=\operatorname{Im}(\Omega)$ as a function of $\boldsymbol{q}$. For this set of parameters, the maximum growth rate $\gamma_{\max }=0.12 \omega_{c i}$ is obtained for $\boldsymbol{q}=\boldsymbol{q}_{\max } \approx k_{x}(\hat{\boldsymbol{x}} \pm \hat{\boldsymbol{y}}) \pm\left(2 \pi / \lambda_{i}\right) \times 0.68 \hat{z}$. The instability is not purely growing and $\operatorname{Re}(\Omega) \approx 0.1 \omega_{c i}$ at $\boldsymbol{q}=\boldsymbol{q}_{\text {max }}$. For the considered pump amplitude, the parametric instability can be characterized as a modified decay instability, i.e., both the up-shifted and down-shifted sidebands are excited while the DA wave is non-resonant $\left(\Omega \neq \Omega_{q}\right)$. As seen in Fig. $1, \gamma$ is large only for $\boldsymbol{q}_{\perp} \approx k_{x} \sim \rho_{S}^{-1} \gg \lambda_{e}^{-1}$ and the excited DA waves can therefore be characterized as driven modified convective cells rather than inertial Alfvén waves.

The evolution of the wave field in the initial phase of the instability can be described as follows: The excited DA waves propagate in the $x$ direction. Due to symmetry, the DA waves set up a standing wave pattern in the $y$ and $z$ direction. The LH wave field is initially dominated by the pump wave but the up-shifted (down-shifted) sideband will introduce perturbations with frequency $\omega_{+}=\omega_{k}+\Omega\left(\omega_{-}=\omega_{k}-\Omega\right)$ and wavevector $\boldsymbol{k}_{+} \approx 2 k_{x} \hat{\boldsymbol{x}} \pm k_{x} \hat{\boldsymbol{y}} \pm q_{z} \hat{z}\left(\boldsymbol{k}_{-} \approx \pm k_{x} \hat{\boldsymbol{y}} \pm q_{z} \hat{z}\right)$.

\section{Structure formation in the nonlinear stage of the parametric instability}

The analysis presented above gives a clear picture of the initial stage of the instability. It explains how the electrostatic part of the hiss, which is modeled by the LH pump wave, can initiate growth of quasineutral density perturbations in the form of driven DA waves. However, when the amplitude of the sidebands $\phi_{ \pm} \exp \left\{i\left[(\boldsymbol{k} \pm \boldsymbol{q}) \cdot \boldsymbol{x}-\left(\omega_{\boldsymbol{k}} \pm \Omega\right) t\right]\right\}$ have grown sufficiently large, additional frequency and wavevector components are excited as a result of scattering off $\eta \neq 0$. This broadening of the wave spectrum is the onset of structure formation and Eq. (7) is no longer sufficient for describing
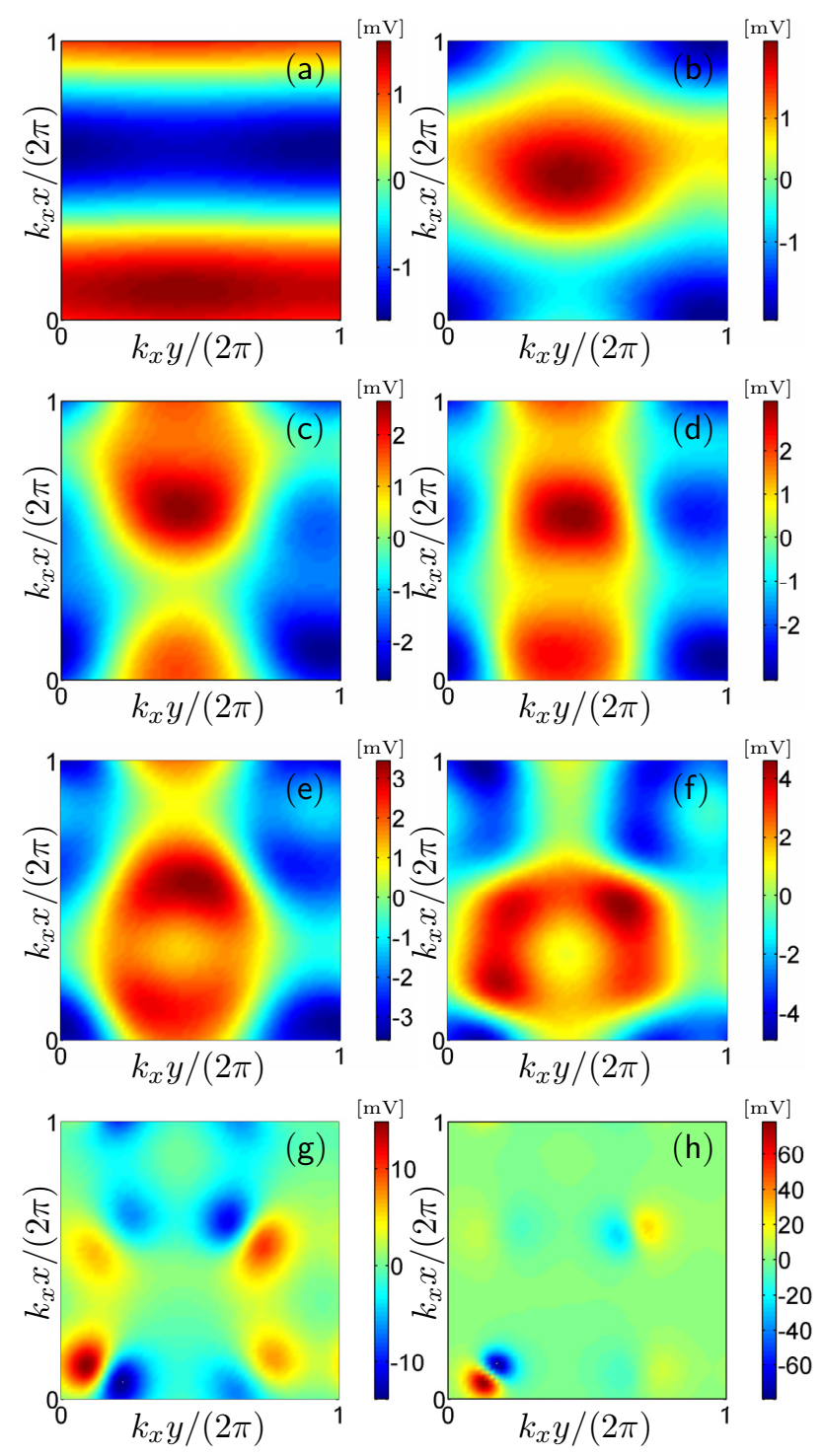

Fig. 2. Panels (a-h) show $\operatorname{Re}\left(\phi_{L}\right)$ in a plane perpendicular to $\boldsymbol{B}_{0}$ at $t=0.51,0.61,0.67,0.69,0.70,0.71,0.72$, and $0.73 \mathrm{~s}$, respectively.

the physics. In order to investigate the structure formation in the nonlinear stage of the modified decay instability, the fully nonlinear three-dimensional Eqs. (1-3) are solved numerically. A pseudo-spectral method is used to approximate the spatial derivatives on a $N_{x} \times N_{y} \times N_{z}=64 \times 64 \times 128$ spatial grid and the solution is advanced in time using a standard fourth order Runge-Kutta method. The pseudo-spectral method implicitly imposes periodic boundary conditions and the size of the simulation box is chosen to be equal to the wave length of the fastest growing mode, i.e., the size of the box is $L_{x}=L_{y}=2 \pi / q_{\perp \max }$ and $L_{z}=2 \pi / q_{z \max }$. A monochromatic LH wave with the wavevector $k=2 \pi /\left(1.7 \rho_{s}\right) \hat{\boldsymbol{x}} \mathrm{m}^{-1}$ and amplitude $E_{0}=6 \mathrm{mV} / \mathrm{m}$ is given as an initial condition. For the DA wave, low amplitude noise is given as an initial condition. 
Figures 2 and 3 display the result of the numerical solution. Figure 2 shows the time evolution of $\operatorname{Re}\left(\phi_{L}\right)$ in the $x-y$ plane for a fix $z$, as discussed later. Figure $2 \mathrm{a}-\mathrm{d}$ shows the initial stage of the modified decay instability, where the evolution of $\phi_{L}$ is fully captured by the analysis in the previous section. In particular, notice the presence of the wavevectors $\boldsymbol{k}_{ \pm}$in the LH wave field. At $t \approx 0.69 \mathrm{~s}$ (Fig. $2 \mathrm{~d}$ ), the amplitudes of the sidebands are of the order of $\phi_{0}$. At this stage, $\eta$ is a sinusoidal perturbation with finite amplitude. The sidebands are subsequently scattered off $\eta$ and generates additional down-shifted frequency components. This cascade to lower frequencies can eventually generate waves with $\omega<\omega_{\mathrm{LH}}$, which are trapped inside the density depletions. This self-localization of $\phi_{L H}$ can be seen in Fig. $2 \mathrm{e}-\mathrm{h}$. Figure 3 shows the time evolution of $\eta$ in the $x-y$ plane for the same $z$ coordinate as in Fig. 2 . At $t \approx 0.72 \mathrm{~s}$, cylindrically symmetric density cavities have formed. The non-localized sinusoidal density perturbations generated in the linear phase of the instability have steepened by the cascade process to form localized structures. The cylindrical density structures are not stationary; the width of the cavity is decreasing while the depth is increasing with time. At $t=0.73 \mathrm{~s}$, the perpendicular width of the cavity is $L_{\perp} \sim 0.3 \rho_{S} \approx 2.5 \mathrm{~m}$ and the depth is $\eta_{\max } \approx 1.5 \%$. Later on in the simulation, the transverse and parallel size of the cavity becomes of the order of the numerical grid size. In Fig. 2 we show a $z$-value near the center of the collapsing structure. The timescale of the collapse is $T_{c} \sim 60 \mathrm{~ms}$. Throughout the collapse ( $t>0.72 \mathrm{~s}$ ), the LH wave field inside the cylindrical cavity is of the form $\phi_{L H}=R\left(r_{\perp}, t\right) \exp \left[-i \varphi-i\left(\omega_{L H}-\delta \omega\right) t\right]+$ c.c., where $\delta \omega>0$ is the frequency shift due to linear dispersion and nonlinearity, $\varphi$ is the azimuthal angle, and $R$ describes the radial variation. The wave front of the LH field is thus rotating in a left-handed sense with a wave frequency less than $\omega_{L H}$.

\section{Results and discussion}

In the present paper, we consider a possible mechanism for the formation of the LHSS. By numerical solutions of the governing equations, including the electron thermal effects introduced by Hall et al. (2005), we show that a plane LH wave can undergo self-localization in cylindrical density cavities formed by the interaction of the LH wave with initially small amplitude DA wave perturbations. We follow the time evolution from the initial phase of the modified decay instability to the formation of cylindrically symmetric density cavities. The initial plane $\mathrm{LH}$ wave with $\omega>\omega_{L H}$ is, by a cascade to lower frequencies, transformed into a localized lefthanded rotating wave with $\omega<\omega_{L H}$. The low-frequency dynamics is essentially electrostatic and can be characterized as driven modified convective cells. The above mentioned features of the numerical solutions are in qualitative agreement with spacecraft observations of LHSS. While the parameters we have used are derived from an observation in the upper
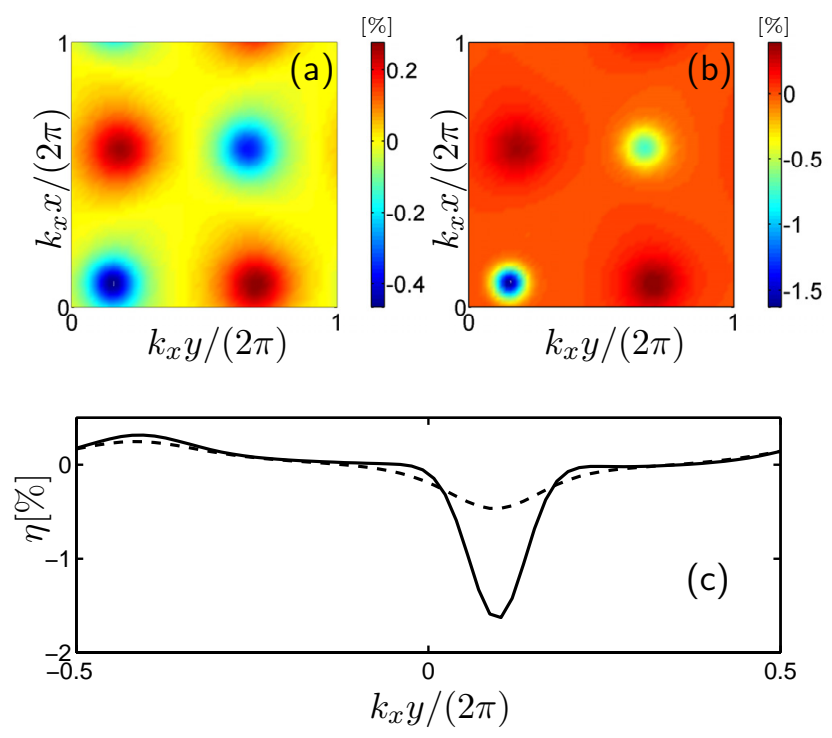

Fig. 3. Panels (a) and (b) show $\eta$ in a plane perpendicular to $\boldsymbol{B}_{0}$ at $t=0.72 \mathrm{~s}$ and $t=0.73 \mathrm{~s}$, respectively. Panel (c) shows $\eta=\eta(y)$ for $k_{x} x /(2 \pi)=0.1$. The dashed (solid) line corresponds to $t=0.72 \mathrm{~s}$ $(t=0.73 \mathrm{~s})$.

ionosphere by the Freja satellite, we showed in Table 1 that the same physics apply also to LHSS observed at higher altitudes.

Our study extends on previous work in several ways. The study of LHSS evolution by Seyler (1994) started from preexisting cylindrical density cavities of order $1 \%$, which is the amplitude range at which most LHSS are actually observed to end up in the upper ionosphere (Pécseli et al., 1996; Dovner et al., 1997). We instead start from infinitesimal dispersive Alfvén waves as initial density perturbations. This approach is similar to the route taken by Shapiro (1998), Shapiro et al. (2003), Ücer and Shapiro (2004), and Ücer and Shapiro (2005), though we follow the evolution of the instability from its initial parametric stage into the subsequent nonlinear evolution. Finally, we include the electron thermal effects. As these were shown by Hall et al. (2005) to substantially narrow the spectrum of the collapsing density fluctuation and to decrease the growth rate of the initial instability to roughly half the value calculated by Shapiro et al. (2003) and Ücer and Shapiro (2005), our study can be considered the first numerically accurate investigation of LHSS formation by DA/LH wave interaction.

No stationary stage is observed in the simulation and the cavities collapse rapidly to small spatial scales, along as well as transverse to the magnetic field, in contrast to observations (Pécseli et al., 1996) but in agreement with previous theoretical and numerical work (Seyler, 1994). However, the considered model is derived from fluid theory, so waveparticle interaction, which requires a kinetic treatment, is neglected. It is likely that kinetic effects like transit-time 
damping (Robinson, 1991) and resonant absorption (Shapiro et al., 1993), leading to e.g. ion transverse heating (Knudsen et al., 2004), set in before the collapse is complete. An interesting question not explicitly addressed in the present work is if the inclusion of electron thermal effects introduces a preferred formation scale size transverse to the magnetic field on the order of the ion sound gyroradius, $\rho_{s}$, as LHSS typically show a width of this order (Knudsen et al., 2004; Tjulin et al., 2004a). However, as the collapse in our simulations proceeds through all scales and no stationary stage is reached, we believe that the perpendicular size is determined by the processes leading to arrest of the collapse rather than by the formation mechanisms studied here. Nevertheless, a parametric study, varying the ratio of $\rho_{s}$ to $\lambda_{e}$, could be suggested to investigate this possibility in future work.

Our model is thus only applicable to the initial stage of LHSS formation, and the derived timescale should be taken a timescale to form an LHSS by the suggested mechanism, not as its complete lifetime. Further, we have used the electrostatic approximation to the describe the LH waves, although it is shown in Hall et al. (2004) that localized LH oscillations with $\omega<\omega_{L H}$ are linearly coupled to the electromagnetic fast magnetosonic wave. As a result of this coupling, wave energy from the localized LH oscillations can leak out from the cavity and damp the localized oscillations. A theory with these extensions may provide a description of a stationary state.

In conclusion, our investigation show that parametric interaction between LH waves in the ambient hiss and DA waves is a likely mechanism for the excitation of LHSS.

Acknowledgements. Topical Editor I. A. Daglis thanks two anonymous referees for their help in evaluating this paper.

\section{References}

Bonnell, J., Schuck, P. W., Pincon, J.-L., Seyler, C. E., and Kintner, P.: Observation of bound states and counterrotating lower hybrid eigenmodes in the auroral ionosphere, Phys. Rev. Lett., 80, 5734-5737, 1998.

Dovner, P. O., Eriksson, A. I., Boström, R., Holback, B., Waldemark, J., Eliasson, L., and Boehm, M.: The occurrence of lower hybrid cavities in the upper ionosphere, Geophys. Res. Lett., 24, 619-622, 1997.

Eriksson, A. I., Holback, B., Dovner, P. O., Boström, R., André, M., Eliasson, L., and Kintner, P. M.: Freja observations of correlated small-scale density depletions and enhanced lower hybrid waves, Geophys. Res. Lett., 21, 1843-1846, 1994.

Hall, J. O.: Conversion of localized lower hybrid oscillations and fast magnetosonic waves at a plasma density cavity, Phys. Plasmas, 11, 5341-5349, 2004.

Hall, J. O., Eriksson, A. I., and Leyser, T. B.: Excitation of localized rotating waves in plasma density cavities by scattering of fast magnetosonic waves, Phys. Rev. Lett., 92, 255002, doi:10.1103/PhysRevLett.92.255002, 2004.

Hall, J. O., Shukla, P. K., and Eliasson, B.: Structure formation by modulational interaction between lower-hybrid and dispersive Alfvén waves, Phys. Plasmas, 12, 052310, doi:10.1063/1.1896373, 2005.

Kintner, P. M., Vago, J., Chesney, S., Arnoldy, R. L., Lynch, K. A., Pollock, C. J., and Moore, T. E.: Localized lower hybrid acceleration of ionospheric plasma, Phys. Rev. Lett., 68, 2448-2451, 1992.

Knudsen, D. J., Wallis, D. D., and James, H. G.: Tethered two-point measurements of solitary auroral density cavities, Geophys. Res. Lett., 26, 2933-2936, 1999.

Knudsen, D. J., Bock, B. J. J., Bounds, S. R., Burchill, J. K., Clemmons, J. H., Curtis, J., Eriksson, A. I., Koepke, M. E., Pfaff, R. F., Reynolds, E. W., Strele, D., Wallis, D. D., and Whaley, N.: Lower-hybrid cavity density depletions as a result of transverse ion acceleration localized on the gyroradius scale, J. Geophys. Res., 109, A04212, doi:10.1029/2003JA010089, 2004.

LaBelle, J., Kintner, P. M., Yau, A. W., and Whalen, B. A.: Large amplitude wave packets observed in the ionosphere in association with transverse ion-acceleration, J. Geophys. Res., 91, 7113-7118, 1986.

Lynch, K. A., Arnoldy, R. L., Kintner, P., Schuck, P., Bonnell, J., and Coffey, V.: Auroral ion acceleration from lower hybrid solitary structures: A summary of sounding rocket observations, J. Geophys. Res., 104, 28515-28534, 1999.

Pécseli, H. L., Iranpour, K., Holter, Ø., Lybekk, B., Holtet, J., Trulsen, J., Eriksson, A., and Holback, B.: Lower hybrid wave cavities detected by the Freja satellite, J. Geophys. Res., 101, 5299-5316, 1996.

Pinçon, J.-L., Kintner, P. M., Schuck, P. W., and Seyler, C. E.: Observation and analysis of lower hybrid solitary structures as rotating eigenmodes, J. Geophys. Res., 102, 17283-17296, 1997.

Robinson, P. A.: Transit-time damping and the arrest of wave collapse, Phys. Fluids B, 3, 545-554, 1991.

Sazhin, S. S., Bullough, K., and Hayakawa, M.: Auroral hiss: a review, Planet. Space Sci., 41, 153-166, 1993.

Schuck, P. W., Seyler, C. E., Pinçon, J.-L., Bonnell, J. W., and Kintner, P. M.: Theory, simulations, and observation of discrete eigenmodes associated with lower hybrid solitary structures, J. Geophys. Res., 103, 6935-6953, 1998.

Schuck, P. W., Bonnell, J., and Kintner, P.: A review of lower hybrid solitary structures, IEEE Trans. Plasma. Sci., 31, 1125, doi:10.1109/TPS.2003.822043, 2003.

Seyler, C. E.: Lower hybrid wave phenomena associated with density depletions, J. Geophys. Res., 99, 19513-19525, 1994.

Shapiro, V. D.: Modulational interaction of lower-hybrid waves with a kinetic-alfvén mode, Phys. Rev. Lett., 81, 3415-3418, 1998.

Shapiro, V. D., Shevchenko, V. I., Solov'ev, G. I., Kalinin, V. P., Bingham, R., Sagdeev, R. Z., Ashor-Abdalla, M., Dawson, J., and Su, J. J.: Wave collapse at the lower-hybrid resonance, Phys. Fluids B, 5, 3148-3162, 1993.

Shapiro, V. D., Ücer, D., and Quest, K. B.: Excitation of inertial alfvén waves via modulational interaction with lowver hybrid waves, Plasma Phys. Rep., 29, 594-605, 2003.

Shukla, P. K. and Stenflo, L., Nonlinear Phenomena Involving Dispersive Alfvén Waves, in: Nonlinear Waves and Turbulence, Lecture Notes in Physics, edited by: Passot, T. and Sulem, P.L., pp. 1-30, Springer Verlag, Berlin, 1999.

Shukla, P. K., Spatschek, K. H., and Balescu, R.: Nonlinear convective cell equations and dipole vortex solutions in homogeneous 
magnetoplasmas, Phys. Lett. A, 107, 461-464, 1985.

Tjulin, A., Eriksson, A. I., and André, M.: Lower hybrid cavities in the inner magnetosphere, Geophys. Res. Lett., 30, 1364, doi:10.1029/2003GL016915, 2003.

Tjulin, A., André, M., Eriksson, A. I., and Maksimovic, M.: Observations of lower hybrid cavities in the inner magnetosphere by the Cluster and Viking satellites, Ann. Geophys., 22, 29612972, 2004a, http://www.ann-geophys.net/22/2961/2004/.
Tjulin, A., Eriksson, A. I., and André, M.: Localization of wave fields in lower hybrid cavities, Ann. Geophys., 22, 2951-2959, 2004b, http://www.ann-geophys.net/22/2951/2004/.

Ücer, D. and Shapiro, V. D.: Alfvén soliton created by reynolds' stress of the lower-hybrid waves, Phys. Lett. A, 328, 196-200, 2004.

Ücer, D., and Shapiro, V. D.: Modulational interaction between short-wavelength lower-hybrid waves and slow, largescale density fluctuations, Phys. Plasmas, 12, 112312, doi:10.1063/1.2135770, 2005. 